\title{
Fragmentary Fuzzy Pseudometrics: Basics of the Theory and Applications in Combinatorics on Words
}

\author{
Raivis BĒTS and Alexander ŠOSTAK \\ Department of Mathematics, University of Latvia, Zelllu street 25, Latvia \\ raivis.bets@lu.lv, sostaks@latnet.lv
}

\begin{abstract}
By modifying the well-known concept of a fuzzy metric introduced in 1994 by A. George and P. Veeramani, we define the notion of a fragmentary fuzzy pseudometric. In this paper, we study basic properties of fragmentary fuzzy pseudometrics, describe the induced fuzzy supratopologies and fuzzy topologies, as well apply fragmentary fuzzy pseudometrics for the description of the analytic structure of the sets of right-infinite words.
\end{abstract}

Keywords: fuzzy metrics, fragmentary fuzzy pseudometrics, fuzzy topologies, right-infinite words

\section{Introduction}

Recently some researchers working in the theory of automatic sequences, in particular, in stringology, became interested in the use of different analytical methods for the study of the structure of sets of infinite words and languages. In particular, different metrics describing distance between infinite words, limits of sequences of words and topologies, both metrizable and non-metrizable, on the set of infinite words were studied. However, in our opinion that we will try to justify in sections 2.2 and 2.4 , the existing methods are far from being appropriate for the reflecting the analytic structure of sets of words and languages. In this paper we develop an alternative approach to the study of the analytic structure of the family of infinite words. The principal idea is to use the so called fuzzy pseudometric instead of ordinary metrics. However, fuzzy pseudometrics, as they are defined by (George and Veeramani, 1994), also are not fully appropriate in order to describe the structure of sets of infinite words. Therefore we revise the "classical" definition of a fuzzy pseudometric by weakening one of its axioms and thus come to the concept of a fragmentary fuzzy pseudometric; the use of the adjective "fragmentary" will be explained in Remarks 2, 3 and justified in Section 4.1. 
The concept of a fragmentary fuzzy pseudometric, being "invented" as a tool for the study of the set of infinite words, appeared to be of its own interest. Although some properties of fragmentary fuzzy pseudometrics are analogous to the corresponding properties of "ordinary" fuzzy pseudometrics, others may be essentially different. In particular, a fragmentary fuzzy pseudometric generally induce a supratopology and not an ordinary topology, as fuzzy pseudometrics do. Being interested in the concept of a fragmentary fuzzy pseudometric itself, we develop basics of the theory of a fragmentary fuzzy pseudometrics in the first part of the paper and then apply it to the case of the set of infinite words.

The structure of the paper is as follows. In the second section we give a very brief introduction into the theory of metric and topological spaces and describe some known metrics on the set of infinite words. We discuss the shortages of ordinary metrics and topologies for the adequate description of their analytic structure of the set of all infinite words. Further, we recall here the concept of a $t$-normed used throughhout the paper and the notion of a fuzzy pseudometric as it is defined by A. George and P. Veeramani, which was the "starting point" for our concept of a fragmentary fuzzy pseudometric introduced and studied in Section 3. This section, being one of the main parts of the work, presents an introduction into the theory of fragmentary fuzzy pseudometrics. Besides, we describe here the (supra)topological structure induced by a fragmentary fuzzy pseudometric. In the 4th section, we develop the construction of a special fuzzy metric on the set of right-infinite words. We illustrate all possible shapes such fragmentary fuzzy metrics depending on the three-element long prefixes of the words. In 5th section we consider the concept a principal fragmentary fuzzy metric and show that the fragmentary fuzzy pseudometrics concstructed in Section 4 are pricipal. This fact allows to get a more clear idea on the topological structure induced on the set of infinite words by fragmentary fuzzy metrics. In the last, $6^{\text {th }}$ section we summarize the main results of this work as well as discuss some prospective directions for the work in this field.

\section{Preliminaries}

\subsection{Introduction to (pseudo-)metrics and (pseudo-)metric spaces}

Recall (see e.g. Frechet, 1906, Engelking, 1977, Kelly, 1955) that a pseudo-metric on a set $X$ is a mapping $d: X \times X \rightarrow[0, \infty)$ such that for all $x, y, z \in X$ :

(1d) $d(x, y)=0 \Longleftarrow x=y$;

(2d) $d(x, y)=d(y, x)$;

(3d) $d(x, z) \leq d(x, y)+d(y, z)$.

In case axiom (1d) is replaced by a stronger axiom

$\left(1^{\prime} \mathrm{d}\right) d(x, y)=0 \Longleftrightarrow x=y ;$

we come to the definition of a metric. A pair $(X, d)$ where $X$ is a set and $d$ is a (pseudo)metric on $X$ is called a (pseudo-)metric space.

In case a stronger version of the axiom $(3 \mathrm{~d})$

$\left(3^{u} \mathrm{~d}\right) d(x, z) \leq \max \{d(x, y), d(y, z)\} ;$ 
holds, a (pseudo-)metric is called an ultra(pseudo-)metric, see e.g. (Kelly, 1955).

Clearly, every ultra (pseudo-)metric is a (pseudo-)metric, but not vise-versa: the standard metric on the plane is not an ultrametric.

\subsection{Metrics on the set of infinite words}

In the literature we have found two kinds of metrics (actually they are ultrametrics) on the set of all infinite words. The first one, that we denote here by $\rho$, is defined as follows, see e.g. (Allouche and Shallit, 2003).

Let

$$
x=\left(x_{0}, x_{1}, x_{2}, \ldots x_{n}, \ldots\right) \text { and } y=\left(y_{0}, y_{1}, y_{2}, \ldots y_{n}, \ldots\right)
$$

be infinite words. Then

$$
\rho(x, y)=\left\{\begin{aligned}
0 & \text { if } x=y \\
2^{-n} & \text { otherwise where } n=\min \left\{i: x_{i} \neq y_{i}\right\}
\end{aligned}\right.
$$

We think that this metric does not give satisfactory information about actual "nearnesstype" relations between the words. For example, consider the following three words $x=(1,1,1,1,1,1,1, \ldots), y=(0,1,1,1,1,1,1, \ldots)$ and $z=(0,0,0,0,0,0,0, \ldots)$. Then $\rho(x, y)=\rho(x, z)=1$, that is in the both cases the distance between these infinite words is the largest possible value in the corresponding metric that equals to 1 . Or, if otherwise stated, all information is got by comparing the first digits of the strings. However, in different situations, expert's intuition may say that $x$ in the above example should be estimated "closer" to $y$ than to $z$. Similar to metric $\rho$, is the so called Cantor metric $c: X \times X \rightarrow[0,1]$, see e.g. (Calude et al., 2009) which also fully depends on the first part, that is on the prefix of the word.

Another known metric on the set of infinite words, denoted here by $\sigma$ has a more subtle principle of its definition:

Let $x=\left(x_{0}, x_{1}, x_{2}, \ldots x_{n}, \ldots\right)$ and $y=\left(y_{0}, y_{1}, y_{2}, \ldots y_{n}, \ldots\right)$ be infinite words, and let for a given $i \in \omega$ (where by omega we denote the set of natural numbers including 0 ) the number $\chi_{i}$ be defined by:

$$
\chi_{i}(x, y)= \begin{cases}0 & \text { if } x_{i}=y_{i} \text { where } i \text { is the } i \text {-th coordinate of the word } \\ 1 & \text { if } x_{i} \neq y_{i} \text { where } i \text { is the } i \text {-th coordinate of the word }\end{cases}
$$

Now let

$$
\sigma(x, y)=\sum_{i=0}^{\infty} \frac{1}{2^{i}} \chi_{i}(x, y)
$$

Then one can easily see that $\sigma: X \times X \rightarrow[0,1]$ is an ultrametric on the set of all infinite words. In our opinion $\sigma$ is more adequate for describing nearness of the words, then $\rho$, since it takes into account information about a word on its whole length, but not considers only the information contained in the prefixes of these words. However, this metric also gives an accumulated information about nearness between the words and neglects all concrete details of this information. For example, let $x=(1,0,0,0,0, \ldots)$, $y=(0,1,1,1,1, \ldots)$ and $z=(0,0,0,0,0, \ldots)$. Then $\sigma(y, z)=1$, and $\sigma(x, z)=1$, and 
hence this metric does not take into account the essential difference of these words, but just accumulates all information in a single number.

Anyway, we think that ordinary metrics are not an adequate analytic tool for describing nearness-type relations between infinite words. Therefore instead of ordinary metrics we propose to use the so called fuzzy metrics. In our opinion, that we try to justify in this paper, fuzzy metrics are much more subtle and, if proper defined, will give a more refined information about the nearness-type properties between the infinite words.

\subsection{Introduction to topology and supratopology}

Recall, that a topology on a set $X$ is a family $\mathcal{T}$ of its subsets, that is $\mathcal{T} \subseteq 2^{X}$ such that

1. $X, \emptyset \in \mathcal{T}$

2. $U, V \in \mathcal{T} \Longrightarrow U \cap V \in \mathcal{T}$

3. $\left\{U_{i}: i \in I\right\} \subseteq \mathcal{T} \Longrightarrow \bigcup_{i \in I} U_{i} \in \mathcal{T}$.

A pair $(X, T)$ where $X$ is a set and $\mathcal{T}$ a topology on it is called a topological space.

In case $\mathcal{T}$ is a family of subsets of a set $X$ satisfying the first and the third axioms of the previous definition, then $\mathcal{T}$ is called supratopology on $X$. Thus, a supratopology on $X$ is a family of its subsets containing an empty set, the set $X$ and closed under taking unions.

Let $\rho: X \times X \rightarrow \mathbb{R}^{+}$be a metric on a set $X$ and, given $x_{0} \in X$ and $r \in(0, \infty)$ let $B\left(x_{0}, r\right)=\left\{x \in X: \rho\left(x_{0}, x\right)<r\right\}$ be the openball with center $x_{0}$ and radiuss $r$. Further let

$$
\mathcal{T}_{\rho}=\left\{\mathcal{O} \in 2^{X}: \forall x \in \mathcal{O} \exists B(x, r) \subseteq \mathcal{O}\right\}
$$

Then $\mathcal{T}_{\rho}$ is a topology on $X$; it is called the topology generated by a metric $\rho$, see e.g. (Engelking, 1977).

\subsection{Topologies in words combinatorics}

There were several attempts to study the topological structure on the sets of finite, infinite and both finite and infinite words. The paper (Calude et al., 2009) contains a survey of different attempts to introduce an appropriate topology on such sets. The authors distinguish two different approaches to this problem. The first one is based on topologies induced by metrics similar to the ones considered in Section 2.2, and hence having no more information than the one contained in the corresponding metrics. In particular, topologies defined by metrics $\rho$, and the Cantor metric $c$ are fully determined by prefixes of the corresponding words. The second is based on the partial order which in a natural way can be introduced on the set of infinite words. However, since any natural order on this set is heavily dependent on the starting part, that is the prefix of the word, the resulting topology has a also an typical prefix character. The authors of (Calude et al., 2009) describe different principles of defining meaningful topologies on the set of infinite words and conclude that although some of these principles are not fully restricted to the prefix order of words itself, but still they rely on it quite heavily. In conclusion the authors say "It should be possible to derive far more general principles which apply to 
many more relations between words by changing the intuition about words being read left to right. "We hope that the topology generated by a fragmentary fuzzy metric in Section 3.2 will make a certain contribution to the study of this problem.

\subsection{Fuzzy (pseudo-)metrics}

In $1951 \mathrm{~K}$. Menger has introduced the concept of a statistical metric (Menger, 1951). The theory of statistical metrics was developed, mainly in the second half of the previous century by different authors, see e.g. the monograph (Schweizer and Sklar,1960). Basing on the concept of a statistical metric In (Kramosil and Michalek, 1975) introduced the notion of a fuzzy metric,we call it KM-fuzzy metric. Actually a KM-fuzzy metric is in a certain sense equivalent to the concept of a statistical metric, but there is essential difference is in its definition and in the interpretation. While the statistical metric $F_{x y}(\lambda)$ on a set $X$ is interpreted as "the probability that the obtained distance between points $x, y \in X$ is smaller than $\lambda \in(-\infty,+\infty)$ "... "the fuzzy approach to the notion of a distance follows from the idea that the distance between two points is not an actually existing real number, but it is a fuzzy notion, i.e. the only way which the distance in question is to ascribe some values from $[0,1]$ to various sentences proclaiming something related to distance" (Kramosil and Michalek, 1975).

In (George and Veeramani, 1994, see also George and Veeramani, 1997), the original concept of a fuzzy metric is slightly modified. On one hand this modification allows many natural examples of fuzzy metrics and fuzzy pseudometrics, in particular fuzzy (pseudo-)metrics constructed from ordinary (pseudo-)metrics. On the other hand George - Veeramani's fuzzy metrics are more appropriate for the definition and the study of the induced topological structure. At present the larger part of the research where fuzzy metrics are involved is done in the framework of George-Veeramani's definition of a fuzzy metric. In our work we revise George-Veeramani's definition of a fuzzy (pseudo-)metric by modifying one of its axioms thus coming to a concept that we call a fragmentary fuzzy pseudometric. ${ }^{1}$ In order to recall the concept of a fuzzy pseudometric (in George and Veeramani, 1994 sense) and related results needed in the main text, first we have to define the notion of a $t$-norm that will be used also throughout the paper.

2.5.1 $t$-norms The notion of a $t$-norm was introduced first in (Menger, 1951), and later studied and applied in the research of many authors, see e.g. fundamental monographs (Schweizer and Sklar,1960, Klement et al., 2000).

Definition 1. (Menger, 1951, see also Schweizer and Sklar,1960) A $t$-norm is a binary operation $*:[0,1] \times[0,1] \rightarrow[0,1]$ on the unit interval $[0,1]$ satisfying the following conditions:

(0t) $*$ is monotone: $\alpha \leq \beta \Rightarrow \alpha * \gamma \leq \beta * \gamma \forall \gamma \in[0,1]$;

(1t) $*$ is commutative: $\alpha * \beta=\beta * \alpha$ for all $\alpha, \beta \in[0,1]$;

\footnotetext{
${ }^{1}$ The meaning of the adjective "fragmentary" and the reason to modify the original definition of a fuzzy pseudometric is explained in Remarks 2 and 3.
} 
(2t) $*$ is associative: $(\alpha * \beta) * \gamma=\alpha *(\beta * \gamma)$ for all $\alpha, \beta, \gamma \in[0,1]$;

(3t) $\alpha * 1=\alpha, \quad \alpha * 0=0$ for all $\alpha \in[0,1]$.

A $t$-norm is called left-continuous in case it satisfies the following axiom

(4t) $*$ distributes over arbitrary joins (suprema): $\alpha *\left(\bigvee_{i \in I} \beta_{i}\right)=\bigvee_{i \in I}\left(\alpha * \beta_{i}\right)$ for every $\alpha \in[0,1]$ and for all $\left\{\beta_{i} \mid i \in I\right\} \subseteq[0,1]$,

where $\bigvee$ denotes the operation of taking suprema in $[0,1]$.

Example 1. Among the most important examples of $t$-norms are the following three (see e.g. Schweizer and Sklar,1960, Klement et al., 2000):

- Let $\alpha * \beta:=\alpha \wedge \beta$ where $\wedge$ denotes the operation of taking minimum in [0,1]. It is called the minimum $t$-norm.

- Let $\alpha * \beta:=\alpha \cdot \beta$ be the product. This is the so called product $t$-norm.

- Let $L=[0,1]$ and $\alpha *_{L} \beta:=\max (\alpha+\beta-1,0)$. This is the well-known Łukasiewicz $t$-norm.

Remark 1. It is known that $\wedge$ is the largest $t$-norm, that is for any $t$-norm $*$ and any $\alpha, \beta \in[0,1]$ it holds $\alpha * \beta \leq \alpha \wedge \beta$. In particular, as one can easily verify, $\alpha \wedge \beta \geq$ $\alpha \cdot \beta \geq \alpha *_{L} \beta$ and the inequality is strict if $\alpha, \beta \in(0,1)$.

2.5.2 Fuzzy pseudometrics Let $\mathbb{R}^{+}=(0, \infty), *:[0,1] \times[0,1] \rightarrow[0,1]$ be a $t$-norm and $X$ be a set.

Definition 2. (George and Veeramani, 1994) A fuzzy pseudo-metric on the set $X$ is a pair $(m, *)$, or simply $m$, where $m: X \times X \times \mathbb{R}^{+} \rightarrow(0,1]$, satisfying the following conditions for all $x, y, z \in X$ and all $s, t \in \mathbb{R}^{+}$:

(1FPM) $m(x, y, t)>0$;

(2FPM) $m(x, y, t)=1$ whenever $x=y$;

(3FPM) $m(x, y, t)=m(y, x, t)$;

(4FPM) $m(x, z, t+s) \geq m(x, y, t) * m(y, z, s)$;

(5FPM) $m(x, y,-): \mathbb{R}^{+} \rightarrow[0,1]$ is continuous.

If $(m, *)$ is a fuzzy pseudometric on $X$, then the triple $(X, m, *)$ is a called a fuzzy pseudometric space.

If axiom (2FPM) is replaced by a stronger axiom

$\left(2^{\prime} \mathrm{FPM}\right) x=y \Longleftrightarrow m(x, y, t)=1$

we get definitions of a fuzzy metric, and the corresponding fuzzy metric space.

Note that axiom (4FPM) combined with axiom (2FPM) implies that the fuzzy pseudometric $m(x, y, t)$ is non-decreasing on the third argument.

The following proposition gives the standard construction of a fuzzy metric from a usual metric on the same set: 
Proposition 1. (George and Veeramani, 1994, George and Veeramani, 1997) Let $(X, d)$ be a pseudo metric space. Let $m_{d}$ be the fuzzy subset of $X \times X \times \mathbb{R}^{+}$, that is a mapping $m_{d}: X \times X \times \mathbb{R}^{+} \rightarrow(0,1]$ defined by

$$
m_{d}(x, y, t)=\frac{t}{t+d(x, y)} .
$$

Then $\left(m_{d}, *\right)$ is a fuzzy metric in case $*=\cdot$ is the product $t$-norm.

\section{Fragmentary fuzzy pseudometrics}

\subsection{Fragmentary fuzzy pseudometrics: basic definitions and preliminary results}

As it was said above, we need a certain modified version of Definition 2:

Definition 3. A fragmentary fuzzy pseudo-metric on the set $X$ is a pair $(m, *)$, or simply $m$ where $*$ is a left semicontinuous $t$-norm and $m: X \times X \times \mathbb{R}^{+} \rightarrow(0,1]$ is a mapping satisfying the following conditions for all $x, y, z \in X, s, t \in \mathbb{R}^{+}$:

(1FFPM) $m(x, y, t)>0$

(2FFPM) $m(x, x, t)=\frac{t}{t+1} \geq m(x, y, t)$;

(3FFPM) $m(x, y, t)=m(y, x, t)$;

(4FFPM) $m(x, z, t+s) \geq m(x, y, t) * m(y, z, s)$;

(5FFPM) function $m(x, y,-): \mathbb{R}^{+} \rightarrow[0,1]$ is continuous and non-decreasing.

If $(m, *)$ is a fragmentary fuzzy metric on $X$, then the triple $(X, m, *)$ is a called a fragmentary fuzzy metric space.

Remark 2. Thus axioms (1FFPM), (3FFPM) and (4FFPM) are the same as the axioms (1FPM), (3FPM) and (4FPM) respectively, but the axiom (2FPM) is replaced by the axiom (2FPM).

Since we replaced the axiom (2FPM) by the axiom (2FFPM) we had to strengthen axiom (5FPM) replacing it by axiom (5FFPM). The reason for this is that combination of axioms (2FFM) and (4FFPM) (as different from the combination of axioms (2FPM) and (4FPM)) does not imply that the function $m(x, y,-): \mathbb{R}^{+} \rightarrow[0,1]$ is non-decreasing. Therefore we have to request this important property explicitely by replacing axiom (5FPM) by axiom (5FFPM).

Remark 3. We think it to be reasonable to replace axiom (2FPM) by an axiom (2FFPM) at least for two reasons.

First, as we will see in Section 4.1, just this generalized version of the definition of a fuzzy pseudometric is an appropriate tool for the description of the "distance" between two infinite words; this "distance" is defined inductively from certain fragments of fuzzy pseudometrics. And second, constituting that a "distance" between two equal objects should be fixed for every $t \in \mathbb{R}^{+}=(0, \infty)$ and not to be a subject of some possible evaluation, does not seem to be very natural in the context of defining "distance" by means of fuzzy metrics. Note also that

$$
\lim _{t \rightarrow \infty} m(x, y, t)=1 \text { whenever } x=y
$$

also in case of a fragmentary fuzzy pseudometric. 
Patterned after (Sapena, 2001) we introduce the following important for us concept:

Definition 4. A fragmentary fuzzy pseudometric is called a fragmentary fuzzy ultra pseudometric if for every $x, y, z \in X, t \in \mathbb{R}^{+}$

$$
m(x, y, t) \geq \min \{m(x, z, t), m(z, y, t)\} .
$$

The next definition is "the fragmentary version" of the concept of a strong fuzzy metric (Sapena and Morillas, 2009, Gregori et al., 2010):

Definition 5. A fragmentary fuzzy pseudometric $m$ on $X$ is called strong if, in addition to the properties (1FFPM), (2FFPM), (3FFPM) and (5FFPM), the following modification of axiom (4FFPM) is satisfied

$\left(4^{s} \mathrm{FFPM}\right) m(x, z, t) \geq m(x, y, t) * m(y, z, t)$ for all $x, y, z \in X$ and for all $t>0$.

To justify this adjective "strong" in this definition we show that actually in this context the axiom (4FFPM) can be obtained "for free", that is axiom $\left(4^{s} \mathrm{FFPM}\right)$ is indeed stronger than axiom (4FFPM). This is proved in the next proposition, cf the analogous statement for fuzzy pseudometrics, (Sapena and Morillas, 2009):

Proposition 2. Let a mapping $m: X \times X \rightarrow \mathbb{R}^{+}$satisfy axioms (1FFPM), (2FFPM), (3FFPM), $\left(4^{s} F F P M\right)$ and (5FFPM), Then $m: X \times X \times \mathbb{R}^{+} \rightarrow[0,1]$ is a fragmentary fuzzy pseudometric.

Proof Referring to axioms ( $4^{s}$ FFPM) and (5FFPM) we get the following series of inequalities:

$$
m(x, z, t+s) \geq m(x, y, t+s) * m(y, z, t+s) \geq m(x, y, t) * m(y, z, s),
$$

which holds for any $x, y, z \in X$ and any $t, s \in \mathbb{R}^{+}$.

Definition 6. A fragmentary strong fuzzy pseudometric $m: X \times X \times \mathbb{R}^{+} \rightarrow(0,1]$ is call a fragmentary strong fuzzy ultra pseudometric if

$$
m(x, y, t) \leq m(x, z, t) \wedge m(z, y, t)
$$

for all $x, y, z \in X$, and every $t \in \mathbb{R}^{+}$.

By modifying in a natural way definitions of continuity and strong continuity (for example, see George and Veeramani, 1997 and Gregori et al., 2009b) for mappings of fuzzy (pseudo)metric spaces, see also Remark 3.17 in (Miñana and Šostak, 2015), we come to the following:

Definition 7. If two fragmentary fuzzy pseudometric spaces $\left(X, m, *_{m}\right)$ and $\left(Y, n, *_{n}\right)$ are given, then a mapping $f: X \rightarrow Y$ is called continuous if for every $\varepsilon \in\left(0, \frac{t}{1+t}\right)$, every $x \in X$ and every $t \in \mathbb{R}^{+}$there exist $\delta \in\left(0, \frac{t}{1+t}\right)$ and $s \in \mathbb{R}^{+}$such that $n(f(x), f(y), t)>\frac{t}{1+t}-\varepsilon$ whenever $m(x, y, s)>\frac{t}{1+t}-\delta$. In symbols:

$$
\forall \varepsilon \in\left(0, \frac{t}{1+t}\right), \forall x \in X, \forall t \in \mathbb{R}^{+} \exists \delta \in\left(0, \frac{t}{1+t}\right), \exists s \in \mathbb{R}^{+} \text {such that }
$$




$$
m(x, y, s)>\frac{t}{1+t}-\delta \Longrightarrow n(f(x), f(y), t)>\frac{t}{1+t}-\varepsilon .
$$

In case when $s$ always can be taken equal to $t$, the mapping $f$ will be called strongly continuous.

Patterned after the construction of the standard fuzzy pseudometric from an ordinary pseudometric, see Proposition 1, we propose a construction of the standard fragmentary fuzzy pseudometric:

Proposition 3. Let $(X, d)$ be an pseudometric space and define the mapping $m_{d}: X \times$ $X \times \mathbb{R}^{+} \rightarrow(0,1]$ by

$$
m_{d}(x, y, t)=\frac{t}{t+1+d(x, y)} .
$$

Then $m_{d}(x, y, t)$ is a fragmentary strong fuzzy pseudometric in case of the product t-norm - and hence also in case of the Eukasiewicz t-norm (see Remark 1).

Proof . It is clear that $m_{d}$ satisfies axioms (1FFM), (2FFM) and (3FFM). The continuity of $m_{d}$ is clear and the non-decreasness of $m_{d}$ can be proved straightforward. To show the validity of axiom ( $\left.4^{s} \mathrm{FFM}\right)$, let $x, y, z \in X$. Then, applying the property $d(x, y) \leq$ $d(x, z)+d(z, y)$ which holds for the pseudometric $d$, we easily verify that

$$
\begin{gathered}
m_{d}(x, y, t)=\frac{t}{t+1+d(x, z)} \geq \\
\frac{t}{t+1+d(x, y)} \cdot \frac{t}{t+1+d(y, z)}=m_{d}(x, z, t) \cdot m_{d}(z, y, t) .
\end{gathered}
$$

Proposition 4. Let $(X, d)$ be an ultra pseudometric space and define the mapping $m_{d}$ : $X \times X \times \mathbb{R}^{+} \rightarrow(0,1]$ by

$$
m_{d}(x, y, t)=\frac{t}{t+1+d(x, y)} .
$$

Then $m_{d}(x, y, t)$ is a fragmentary strong fuzzy ultra pseudo-metric in case of the t-norm $\wedge$ and hence (by Remark 1 also in case of any other t-norm.

Proof As in the case of Proposition 3, it is clear that $m_{d}$ satisfies axioms (1FFM), (2FFM) and (3FFM). The continuity of $m_{d}$ is clear and the non-decreasness of $m_{d}$ can be proved straightforward. Noticing that the axiom $d(x, y) \leq \max \{d(x, z), d(z, y)\}$ of the ultra pseudometric $d$ implies the inequality

$$
\frac{t}{t+1+d(x, z)} \geq \frac{t}{t+1+d(x, y)} \wedge \frac{t}{t+1+d(y, z)},
$$

we conclude that $m_{d}(x, z) \geq m_{d}(x, y) \wedge m_{d}(y, z)$.

In (Gregori and Romaguera, 2004) a fuzzy pseudo-metric $m$ on $X$ is called stationary, if $m$ does not depend on $t$, i.e. if for every $x, y \in X$, the function $m_{x, y}(t)=$ $m(x, y, t)$ is constant. We will need the following specification of this property.

Definition 8. A fragmentary fuzzy metric $m$ on $X$ is said to be stationary on the interval $[c, d] \subseteq \mathbb{R}^{+}$, if for each $x, y \in X$, the function $m_{x, y}(t)=m(x, y, t)$ is constant on $[c, d]$. 


\subsection{Supratopology and topology induced by a fragmentary fuzzy pseudometric}

Let $m: X \times X \times \mathbb{R}^{+} \rightarrow(0,1]$ be a fragmetary fuzzy pseudometric. We follow the lines of the construction of a topology from a fuzzy metric, developed back in (George and Veeramani, 1994) in order to define the topology induced by a fragmentary fuzzy metric. However, since the axiom

(2FPM) $x=y \Longrightarrow m(x, y, t)=1 \forall x, y \in X, \forall t \in \mathbb{R}^{+}$

of the fuzzy pseudometric, in case of a fragmentary fuzzy pseudometric is replaced by the axiom

(2FFPM) $m(x, x, t)=\frac{t}{1+t} \geq m(x, y, t) \forall x, y \in X, \forall t \in \mathbb{R}^{+}$, we have to made essential modification in the construction.

Given a point $x \in X$, and $t \in \mathbb{R}^{+}$we define the "ball" with center $x$, at the level $t$ and radiuss $\varepsilon \in\left(0, \frac{t}{1+t}\right)$ as follows:

$$
B(x, \varepsilon, t)=\left\{y \in X: m(x, y, t)>\frac{t}{1+t}-\varepsilon\right\} .
$$

Note that

$$
t \leq s \Longrightarrow B(x, \varepsilon, t) \subseteq B(x, \varepsilon, s) \text { and } \varepsilon \leq \delta \Longrightarrow B(x, \varepsilon, t) \subseteq B(x, \delta, t) .
$$

We use the family of balls

$$
\mathfrak{B}=\left\{B(x, \varepsilon, t) \mid x \in X, t \in \mathbb{R}^{+}, \varepsilon \in\left(0, \frac{t}{1+t}\right)\right\}
$$

to induce the family $T_{m}$ of "open" subsets of the set $X$ :

$$
T_{m}=\left\{\mathcal{O} \in 2^{X}: \forall x \in X \exists t \in \mathbb{R}^{+}, \exists \varepsilon \in\left(0, \frac{t}{1+t}\right) \text { such that } B(x, \varepsilon, t) \subseteq \mathcal{O}\right\} .
$$

In (George and Veeramani, 1994, George and Veeramani, 1997) it is proved that in case of a fuzzy pseudometric $m$ the family

$$
\mathfrak{B}^{*}=\{B(x, \varepsilon, t) \mid x \in X, t \in(0, \infty), \varepsilon \in(0,1)\},
$$

defined in an analogous way, satisfies necessary conditions to be a base for the topology $T_{m}$ on $X$. We cannot prove an analogous theorem in our case, since the family $\mathfrak{B}$ (as different from the family $\mathfrak{B}^{*}$ ) contains only "balls" with sufficiently "small" radiuss $\varepsilon \in$ $\left(0, \frac{t}{1+t}\right)$. When reasoning patterned after Section 3 in (George and Veeramani, 1994), we cannot guarantee that for every $y \in B(x, \varepsilon, t)$ there exists a ball $\mathcal{B}(y, \delta, s)$ such that $B(y, \delta, s) \subseteq B(x, \varepsilon, t)$. Therefore the collection of all "balls"

$$
\mathfrak{B}=\left\{B(x, \varepsilon, t) \mid x \in X, t \in(0, \infty), \varepsilon \in\left(0, \frac{t}{1+t}\right)\right\}
$$

generally does not satisfy the criteria to be a base for a topology. Hence by taking arbitrary unions of the balls from the family $\mathfrak{B}$ we obtain a supratopology $T_{m}$, but generally 
not a topology. We call it a supratopology induced by fragmentary fuzzy pseudometric $m .^{2}$

Fortunately, in case of a fragmentary fuzzy strong ultra pseudometric, that is the most important for our principal merits case the induced supratopology is indeed a topology. We show this in the following Theorem:

Theorem 1. Let $m: X \times X \times \times \mathbb{R}^{+} \rightarrow(0,1]$ be an fragmentary fuzzy ultra pseudometric. Then for every $y \in B(x, \varepsilon, t)$ it holds $B(y, \varepsilon, t) \subseteq B(x, \varepsilon, t)$.

Proof To show that $B(y, \varepsilon, t) \subseteq B(x, \varepsilon, t)$ let $z \in B(y, \varepsilon, t)$. Then $m(x, y, t)>\frac{t}{1+t}-\varepsilon$ and, since $m$ is fragmentary, $m(x, x, t)=\frac{t}{t+1}$. Now, recalling that $m$ is a fragmentary fuzzy ultra pseudometric we conclude that $m(y, y, t)>\frac{t}{1+t}-\varepsilon$, that is $y \in B(y, \varepsilon, t)$. Now, let $z \in B(y, \varepsilon, t)$. Then

$$
m(x, z, t) \geq m(x, y, t) \geq m(x, y, t) \wedge m(y, z, t) \geq\left(\frac{t}{1+t}-\varepsilon\right) \wedge\left(\frac{t}{1+t}-\varepsilon\right)
$$

and hence $z \in B(x, \varepsilon, t)$.

Further we consider some properties of a supratopology induced by a fragmentary fuzzy strong pseudometric without assumption that it is "ultra'.

One can easily verify the following proposition:

Proposition 5. Assume that $\left(X_{1}, m_{1}, *_{m_{1}}\right)$ and $\left(X_{2}, m_{2}, *_{m_{2}}\right)$ are fragmentary fuzzy pseudometric spaces. Then a mapping

$$
f:\left(X_{1}, m_{1}, *_{m_{1}}\right) \rightarrow\left(X_{2}, m_{2}, *_{m_{2}}\right)
$$

is continuous (in the sense of definition 7) if and only if the mapping of the corresponding supratopological spaces

$$
f:\left(X_{1}, T_{m_{1}}\right) \rightarrow\left(x_{2}, T_{m_{2}}\right)
$$

is continuous (that is $\forall V \in T_{m_{2}} \Rightarrow f^{-1}(V) \in T_{m_{1}}$ ).

Definition 9. Let $(X, m, *)$ be a fragmentary fuzzy pseudometric space and $A \subseteq X$. Then $A$ is called bounded if there exist $t \in(0, \infty)$ and $r \in(0,1)$ such that $m(x, y, t)>$ $\frac{t}{1+t}-r$ for all $x, y \in A$.

We get the following by reasoning in the same way as in the proof of Theorem 3.9 in (George and Veeramani, 1994):

Theorem 2. Every set A which is compact in the supratopology induced by a fragmentary fuzzy pseudometric $m$ is bounded.

The following theorem establishes connections between convergence of sequences in a fragmentary fuzzy pseudometric and their convergence in the induced supratopology.

${ }^{2}$ Of course, we can use the family $\mathfrak{B}$ as a subbase for some topology on $X$. However, we think that it is more natural in this case to remain on the level of a supratopology 
Theorem 3. Let $(X, *, t)$ be a strong fuzzy $\frac{t}{1+t}$-pseudometric space. Then for a sequence $\left(x_{n}\right)_{n \in \mathbb{N}} \subseteq X$, it holds $x_{n} \rightarrow x$ in the supratopology $T_{m}$ if and only if $\lim _{n \rightarrow \infty} m\left(x_{n}, x, t\right)=\frac{t}{1+t}$.

Proof Suppose $x_{n} \rightarrow x$ in the supratopology $T_{m}$. Then for each $t \in \mathbb{R}^{+}$, and for each $\varepsilon \in\left(0, \frac{t}{1+t}\right)$ and $B(x, \varepsilon, t) \neq \emptyset$ there exists $n_{0}$ such that $x_{n} \in B(x, \varepsilon, t)$ for all $n \geq n_{0}$. Hence $m\left(x, x_{n}, t\right) \geq \frac{t}{1+t}-\varepsilon$ for all $n \geq n_{0}$. Since on the other hand, $m\left(x, x_{n}, t\right) \leq \frac{t}{1+t}$, we conclude, that $\lim _{n \rightarrow \infty} m\left(x, x_{n}, t\right)=\frac{t}{1+t}$.

Conversely, assume that $\lim _{n \rightarrow \infty} m\left(x, x_{n}, t\right)=\frac{t}{1+t}$. Then for each $\varepsilon \in\left(0, \frac{t}{1+t}\right)$ there exists $n_{0}$ such that for all $n \geq n_{0}$ it holds $m\left(x_{n}, x, t\right) \geq \frac{t}{1+t}-\varepsilon$. However, this means that $x_{n} \in B(x, \varepsilon, t)$. Since this holds for every $\varepsilon \in\left(0, \frac{t}{1+t}\right)$ that is for every non-empty ball $B(x, \varepsilon, t)$, we conclude that $\lim _{n \rightarrow \infty} x_{n}=x$ in the supratopology $T_{m}$.

\section{Fragmentary fuzzy ultra pseudometric on the set of infinite words}

\subsection{Construction of a fragmentary fuzzy ultra pseudometric on the set of infinite words}

Let $X$ be the set of infinite words. We define a sequence

$$
\left\{d_{n} \mid n \in \mathbb{N} \cup\{0\}\right\}
$$

of ultra pseudometrics on $X$ as follows. Let $x=\left(x_{0}, x_{1}, x_{2}, \ldots\right), y=\left(y_{0}, y_{1}, y_{2}, \ldots\right) \in$ $X$ and let $\chi_{i}(x, y)=0$ if $x_{i}=y_{i}$ and $\chi_{i}(x, y)=1$ if $x_{i} \neq y_{i}$. We define:

$d_{0}(x, y)=\chi_{0}(x, y)$

$d_{1}(x, y)=\chi_{0}(x, y)+\frac{\chi_{1}(x, y)}{2}$

$d_{2}(x, y)=\chi_{0}(x, y)+\frac{\chi_{1}(x, y)}{2}+\frac{\chi_{2}(x, y)}{2^{2}} ;$

$\ddot{d}_{n}(x, y)=\sum_{i=0}^{n} \frac{\chi_{i}(x, y)}{2^{i}}$

. .

Proposition 6. Every $d_{n}$ is an ultra pseudometric.

Proof Obviously every $\frac{\chi_{i}(x, y)}{2^{i}}$ is an ultra pseudo metric. From here we conclude that every $d_{n}(x, y)$ is an ultra pseudo metric by induction referring to the following easily provable Lemma:

Lemma 1. Let $d_{1}, d_{2}: X \times X \rightarrow \mathbb{R}^{+}$be ultra pseudo metrics. Assume that $d_{1}(x, y) \in$ $\{0\} \cup[a, 1]$ for any $x, y \in X$ and that $d_{2}(x, y) \in\left[0, \frac{a}{2}\right]$. Then $d=d_{1}+d_{2}: X \times X \rightarrow$ $[0,1]$ is an ultra pseudometric. 
Basing on this sequence of ultra pseudometrics and referring to Proposition 4 we construct the sequence of fragmentary fuzzy strong ultra pseudometrics on the set $X$ of all right-infinite words:

$$
\begin{aligned}
& \mu_{0}(x, y, t)=\frac{t}{t+1+d_{0}(x, y)} \\
& \mu_{1}(x, y, t)=\frac{t}{t+1+d_{1}(x, y)} \\
& \mu_{2}(x, y, t)=\frac{t}{t+1+d_{2}(x, y)} \\
& \ldots ; \\
& \mu_{n}(x, y, t)=\frac{t}{t+1+d_{n}(x, y)}
\end{aligned}
$$

Further we define the following family of mappings:

$m_{0}(x, y, t)=\mu_{0}(x, y, t)$;

$m_{1}(x, y, t)=\mu_{1}(x, y, t) \vee \mu_{0}(x, y, 1)$

$m_{2}(x, y, t)=\mu_{2}(x, y, t) \vee \mu_{1}(x, y, 2)$;

$\ldots$

$m_{n}(x, y, t)=\mu_{n}(x, y, t) \vee \mu_{n-1}(x, y, n-1)$;

$\ldots$

Proposition 7. Mappings $m_{n}: X \times X \times \mathbb{R}^{+} \rightarrow[0,1]$ are fragmentary strong fuzzy ultra metrics on the set $X$ of infinite words.

Proof From propositions 4 and 6 we know that each $\mu_{n}$ is a fragmentary fuzzy strong ultra pseudometric, that is $\mu_{n}(x, z, t) \geq \mu_{n}(x, y, t) \wedge \mu_{n}(y, z, t)$. Since $m_{n}(x, y, t)=$ $\mu_{n}(x, y, t) \vee a_{n}$ where $a_{n}$ is some constant, it is clear that $m_{n}(x, y, t)$ is a fragmentary fuzzy ultra pseudometric and besides $m_{n}(x, z, t) \geq m_{n}(x, y, t) \wedge m_{n}(y, z, t)$, that is $m_{n}$ is strong.

Finally, we construct a mapping $\mathfrak{m}: X \times X \times \mathbb{R}^{+} \rightarrow(0,1]$ as follows:

$$
\mathfrak{m}(x, y, t)=\left\{\begin{array}{cl}
m_{0}(x, y, t) & \text { if } 0<t \leq 1 \\
m_{1}(x, y, t) & \text { if } 1<t \leq 2 \\
m_{2}(x, y, t) & \text { if } 2<t \leq 3 \\
\cdots & \text { if } n<t \leq n+1 \\
m_{n}(x, y, t) & \text { if }
\end{array}\right.
$$

Theorem 4. The mapping $\mathfrak{m}: X \times X \times \mathbb{R}^{+} \rightarrow[0,1]$ is a fragmentary fuzzy strong ultra pseudometric.

The proof is straightforward from Proposition 7.

\subsection{Possible shapes of the fragmentary fuzzy strong ultra metric. $m$ in the first 3 stages}

We illustrate the shape in the initial interval $(0,3]$ of the constructed fragmentary fuzzy ultra pseudometric $m$ describing the distance between infinite words $x=\left(x_{0} x_{1} x_{2} \ldots\right)$ and $y=\left(y_{0} y_{1}, y_{2} \ldots\right)$ in dependence on the first three values $x_{0}, x_{1}, x_{2}, y_{0}, y_{1}$, and $y_{2}$. 


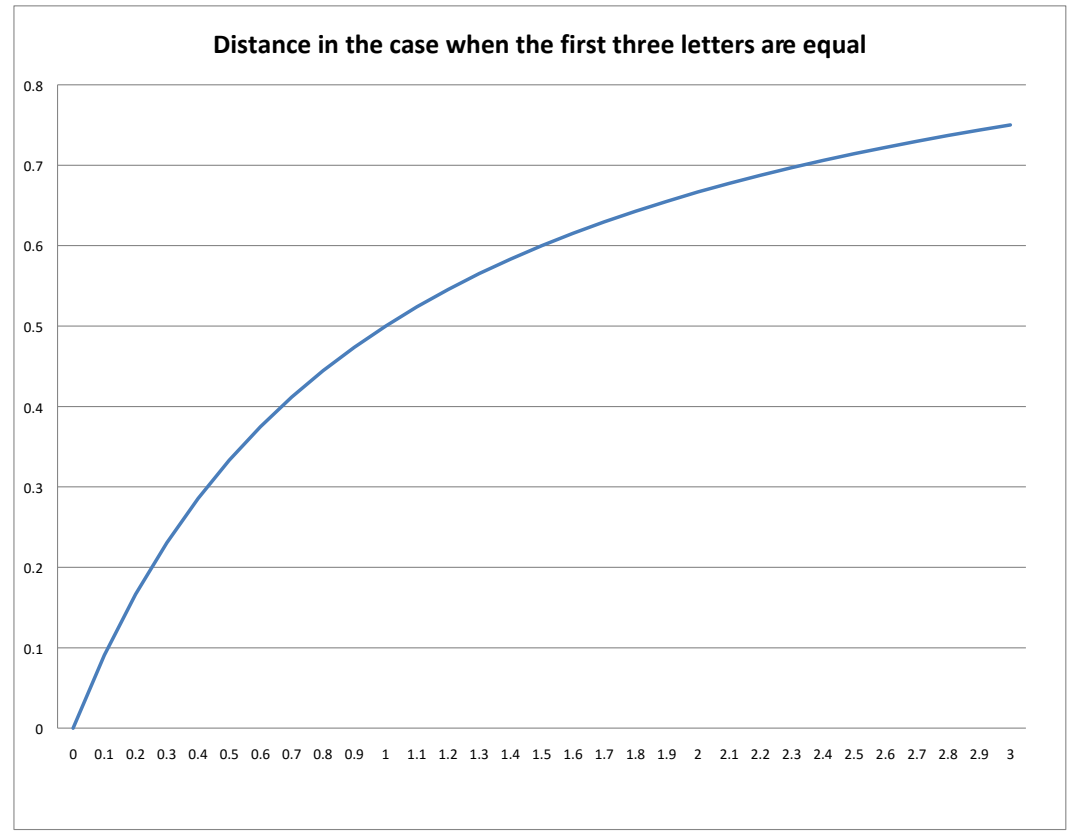

Fig. 1. The metrics for the Case 1

1. The case $x_{0}=y_{0}, x_{1}=y_{1}, x_{2}=y_{2}$. Then

$$
\mathfrak{m}(x, y, t)=\frac{t}{t+1} \text { for } t \in(0,3] .
$$

2. The case $x_{0}=y_{0}, x_{1}=y_{1}, x_{2} \neq y_{2}$. Then

$$
\mathfrak{m}(x, y, t)=\left\{\begin{aligned}
\frac{t}{t+\frac{1}{2}} & \text { if } 0<t \leq 2 \\
\frac{2}{3} & \text { if } 2<t \leq \frac{5}{2} \\
\frac{t}{t+\frac{5}{4}} & \text { if } \frac{5}{2}<t \leq 3
\end{aligned}\right.
$$

3. The case $x_{0}=y_{0}, x_{1} \neq y_{1}, x_{2}=y_{2}$. Then

$$
\mathfrak{m}(x, y, t)=\left\{\begin{aligned}
\frac{t}{t+1} & \text { if } 0<t \leq 1 \\
\frac{1}{2} & \text { if } 1<t \leq \frac{3}{2} \\
\frac{t}{t+\frac{3}{2}} & \text { if } \frac{3}{2}<t \leq 3
\end{aligned}\right.
$$




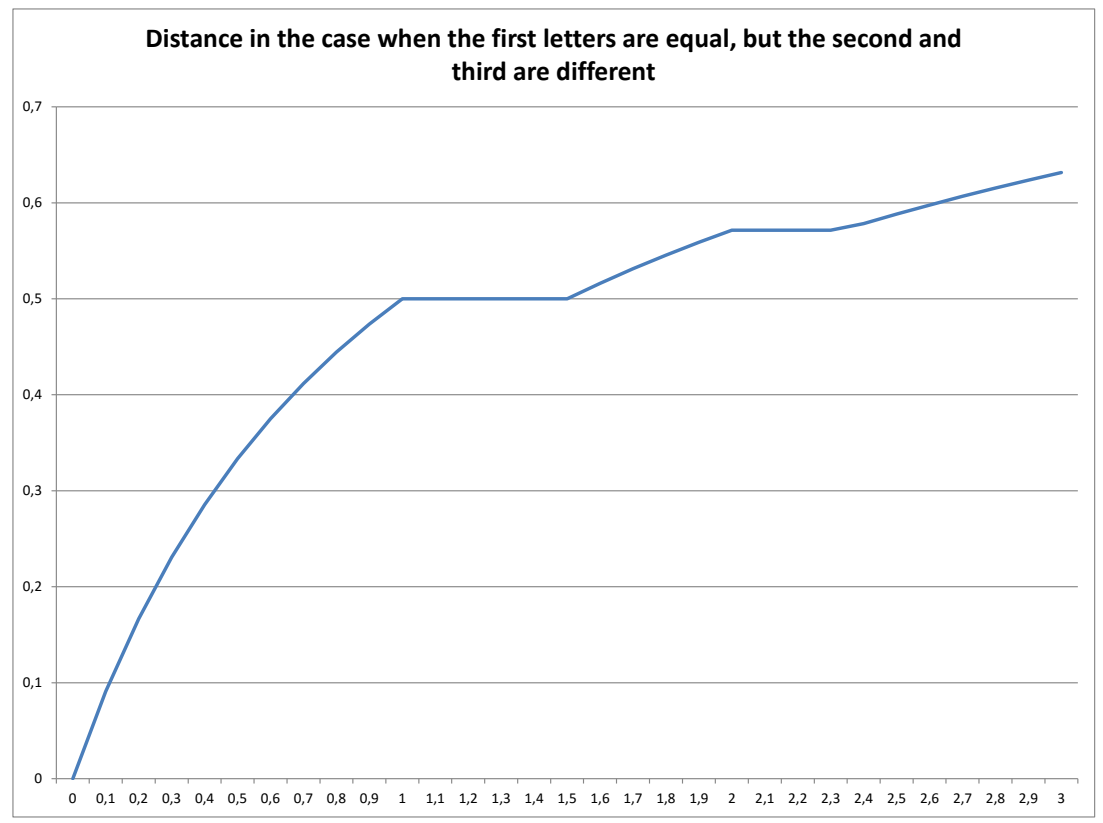

Fig. 2. The metrics for the Case 4

4. The case $x_{0}=y_{0}, x_{1} \neq y_{1}, x_{2} \neq y_{2}$. Then

$$
\mathfrak{m}(x, y, t)=\left\{\begin{aligned}
\frac{t}{t+1} & \text { if } 0<t \leq 1 \\
\frac{1}{2} & \text { if } 1<t \leq \frac{3}{2} \\
\frac{t}{t+\frac{3}{2}} & \text { if } \frac{3}{2}<t \leq 2 \\
\frac{4}{7} & \text { if } 2<t \leq \frac{7}{3} \\
\frac{t}{t+\frac{7}{4}} & \text { if } \frac{7}{3}<t \leq 3
\end{aligned}\right.
$$

5. The case $x_{0} \neq y_{0}, x_{1}=y_{1}, x_{2}=y_{2}$. Then

$$
m(x, y, t)=\frac{t}{t+2} \text { for } t \in(0,3]
$$

6. The case $x_{0} \neq y_{0}, x_{1}=y_{1}, x_{2} \neq y_{2}$. Then

$$
\mathfrak{m}(x, y, t)=\left\{\begin{aligned}
\frac{t}{t+2} & \text { if } 0<t \leq 2 \\
\frac{1}{4} & \text { if } 2<t \leq \frac{9}{4} \\
\frac{t}{t+\frac{9}{4}} & \text { if } \frac{9}{4}<t \leq 3
\end{aligned}\right.
$$




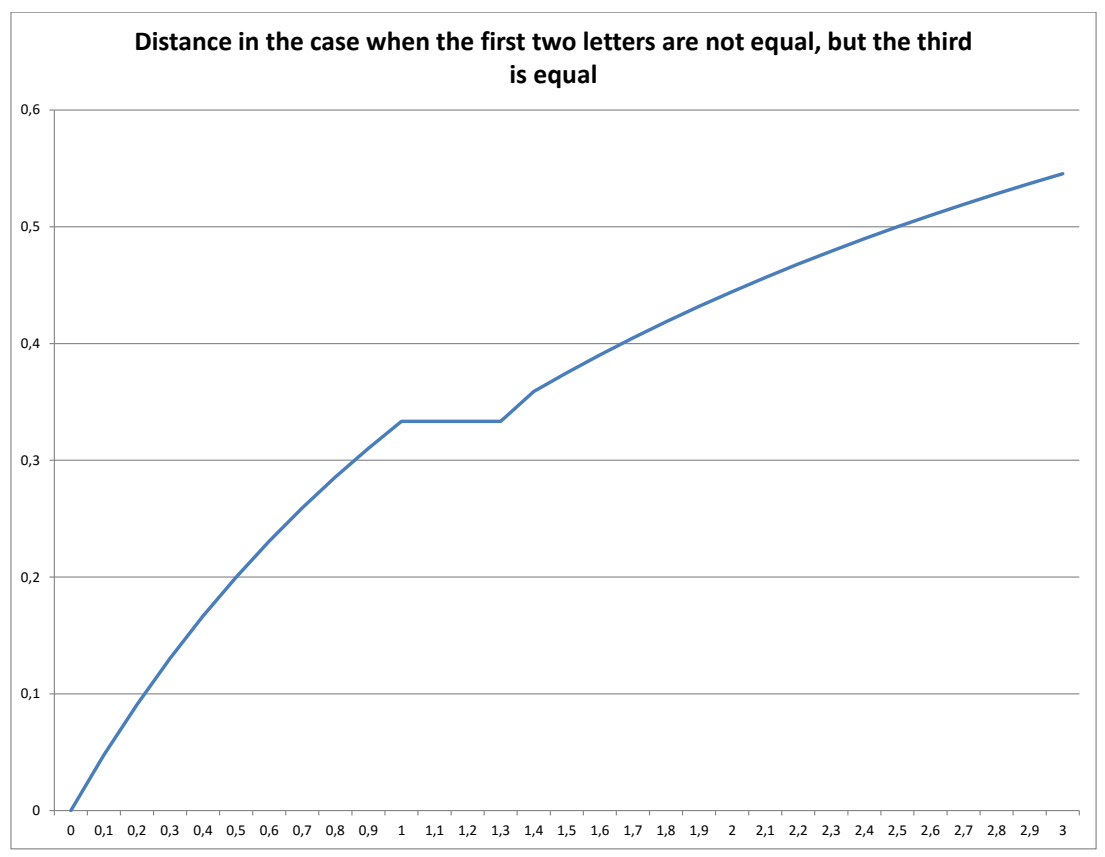

Fig. 3. The metrics for the Case 7

7. The case $x_{0} \neq y_{0}, x_{1} \neq y_{1}, x_{2}=y_{2}$. Then

$$
\mathfrak{m}(x, y, t)=\left\{\begin{aligned}
\frac{t}{t+2} & \text { if } 0<t \leq 1 \\
\frac{1}{3} & \text { if } 1<t \leq \frac{5}{4} \\
\frac{t^{2}}{t+\frac{5}{2}} & \text { if } \frac{5}{2}<t \leq 3
\end{aligned}\right.
$$

8. The case $x_{0} \neq y_{0}, x_{1} \neq y_{1}, x_{2} \neq y_{2}$. Then

$$
\mathfrak{m}(x, y, t)=\left\{\begin{aligned}
& \frac{t}{t+2} \text { if } 0<t \leq 1 \\
& \frac{1}{3} \text { if } 1<t \leq \frac{5}{4} \\
& \frac{t}{t+\frac{5}{2}} \text { if } \frac{5}{4}<t \leq 2 \\
& \frac{\frac{4}{9}}{t+\frac{11}{4}} \text { if } 2<t \leq \frac{11}{5} \\
& \frac{t}{5}<t \leq 3
\end{aligned}\right.
$$

\section{Principal fragmentary fuzzy pseudometrics}

When defining a (supra)topology induced by a fragmentary fuzzy pseudometric, for each $x \in X$, we have to take into consideration all $\varepsilon \in\left(0, \frac{t}{1+t}\right)$ as well as all $t \in \mathbb{R}^{+}$. 
The structure of the topology becomes more lucid and simple if the families

$$
\mathcal{B}_{t}=\left\{B(x, \varepsilon, t): x \in X, \varepsilon \in\left(0, \frac{t}{1+t}\right)\right\}
$$

induce the same topology on the set $X$ for all $t \in \mathbb{R}^{+}$. In other words this means that $\mathcal{B}_{t}=\left\{B(x, \varepsilon, t): \varepsilon \in\left(0, \frac{t}{1+t}\right)\right\}$ is a local base at the point $x$ for the supratopology $T_{m}$. Pattern after (Gregori et al., 2009a) we call such fragmentary fuzzy metrics principle.

For our merits we will need the following specification of this property.

Definition 10. A fragmentary fuzzy pseudometric $m: X \times X \times \mathbb{R}^{+} \rightarrow(0,1]$ is called principal on the interval $[c, d] \subseteq(0, \infty)$ if the families

$$
\mathcal{B}_{t}=\left\{B(x, \varepsilon, t): \varepsilon \in\left(0, \frac{t}{1+t}\right)\right\}
$$

induce the same topology on the set $X$ for all $t \in[c, d]$.

\section{1 "Principality" of the fragmentary fuzzy ultra pseudometric $\mathfrak{m}$ constructed in Section 4.1}

Theorem 5. Fragmentary fuzzy ultra pseudometric $\mathfrak{m}$ on the family of infinite words constructed in Section 4.1 is principal.

To prove this theorem we first establish two lemmas:

Lemma 2. Let $d: X \times X \rightarrow[0,1]$ be a pseudometric and a fuzzy pseudo-metric $m$ : $X \times X \times \mathbb{R}^{+} \rightarrow[0,1]$ be such that $m(x, y, t)=\frac{t}{t+1+d(x, y)}$ for each $t \in[c, d] \subseteq(0, \infty)$. Then the fuzzy pseudometric $m: X \times X \times \mathbb{R}^{+} \rightarrow[0,1]$ is principal on $[c, d]$

To prove this lemma it is sufficient to show that for each $x \in X$, for each $t \in[c, d]$ and for each $\varepsilon \in\left(0, \frac{t}{1+t}\right)$ we can find $\delta \in\left(0, \frac{t}{1+t}\right)$ such that $B(x, \delta, t)=B(x, \varepsilon, c)$.

Then

$$
B(x, \varepsilon, c)=\left\{y: \frac{c}{c+1+d(x, y)}>\frac{t}{1+t}-\varepsilon\right\},
$$

and

$$
B(x, \delta, t)=\left\{y: \frac{t}{t+1+d(x, y)}>\frac{t}{1+t}-\delta\right\} .
$$

Since $t \in[c, d]$ we can find $\alpha \in(0,+\infty)$ such that $t=c+\alpha$. Now the requested condition that

$$
B(x, \delta, t)=B(x, \varepsilon, c)
$$

can be reformulated as follows: For a given $\alpha$ and $\varepsilon$ we must find $\delta \in\left(0, \frac{t}{1+t}\right)$ such that

$$
\frac{c}{c+b}=1-\varepsilon \Longleftrightarrow \frac{c+\alpha}{c+\alpha+b} \text {. }
$$


Making elementary calculations we get from here that

$$
\delta=\frac{\varepsilon t}{t+\alpha(1-\varepsilon)} .
$$

Obviously $\delta=\varepsilon$ when $t=c$ and $\delta$ decreases from $\varepsilon$ to $\frac{\varepsilon t}{t+(d-c)(1-\varepsilon)}$ as $t$ increases from $c$ to $d$.

Lemma 3. If a fragmentary fuzzy pseudometric $m: X \times X \times \mathbb{R}^{+} \rightarrow(0,1]$ is stationary on an interval $[c, d]$, then it is also principal on this interval.

The proof is obvious, since stationarity in this case means that $m(x, y, t)=m(x, y, s)$ for all $t, s \in[c, d]$ and hence topologies generated by all fragmentary fuzzy pseudometrics $m(x, y, t)$, where $t \in[c, d]$, coincide.

\section{Proof of the theorem}

From the construction of the fragramentary fuzzy ultra pseudometric $\mathfrak{m}(x, y, t)$, see Subsection 4.1, see also Subsection 4.2, it is clear that, for a given infinite word $x=$ $\left(x_{o}, x_{1}, x_{2}, \ldots\right)$, this fragmentary fuzzy ultra pseudometric determines one of the following three types of sequences of numbers

$$
\begin{gathered}
0=c_{0}<c_{1}<c_{2}<c_{3} \ldots c_{2 k-1}<c_{2 k}<c_{2 k+1}<\ldots \\
0=c_{0}<c_{1}<c_{2}<c_{3} \ldots c_{2 k-1}<c_{2 k}<c_{2 k+1} \\
0=c_{0}<c_{1}<c_{2}<c_{3} \ldots c_{2 k-1}<c_{2 k}
\end{gathered}
$$

such that on the interval $\left(c_{0}, c_{1}\right]$ and on each interval $\left[c_{2 k}, c_{2 k+1}\right]$ for $k \in \omega$ the fragmentary fuzzy metric $\mathfrak{m}(x, y, t)$ is defined by the formula $\mathfrak{m}(x, y, t)=\frac{t}{t+1+d(x, y)}$ and on each interval $\left[c_{2 k-1}, c_{2 k}\right]$ for $k \in \omega$ the fragmentary fuzzy ultra psdeudometric is stationary.

Consider first the case

$$
0=c_{0}<c_{1}<c_{2}<c_{3} \ldots c_{2 k-1}<c_{2 k}<c_{2 k+1}<\ldots
$$

Referring to Lemma 2 we conclude that the topologies generated by the framentary fuzzy ultra pseudometric $m(x, y, t)$ coincide for all $t \in\left(c_{0}, c_{1}\right]$ and all $t \in\left[c_{2 k}, c_{2 k+1}\right]$, $k \in \omega$. On the other hand, referring to Lemma 3, we see that the topologies generated by the fragmentary fuzzy ultra pseudometric $\mathfrak{m}(x, y, t)$ coincide for all $t \in\left[c_{2 k-1}, c_{2 k}\right]$, $k \in \mathbb{N}$. Since the end points of the intervals belong to the both types of the intervals, by induction we conclude that the topologies generated by all $t \in(0, \infty)$ coincide and hence the fragmentary fuzzy ultra pseudometric is principal.

In case of a finite sequence $0=c_{0}<c_{1}<c_{2}<c_{3} \ldots c_{2 k-1}<c_{2 k}<c_{2 k+1}$ we are reasoning as in the first case and finish the proof noticing that at the last infinite interval $\left(c_{2 k+1}, \infty\right)$ the fragmentary fuzzy ultra pseudometric is stationary.

In case of a finite sequence $0=c_{0}<c_{1}<c_{2}<c_{3} \ldots c_{2 k-1}<c_{2 k}$ we are reasoning as in the first case and finish the proof noticing that at the last infinite interval $\left(c_{2 k+1}, \infty\right)$ the fragmentary fuzzy ultra pseudometric is defined by the formula $m(x, y, t)=\frac{t}{t+1+d(x, y)}$ and hence is principal. 


\section{Conclusions}

In this paper we have introduced the concept of a fragmentary fuzzy pseudometric as a modification of the well-known concept of a fuzzy metric as it is defined in the paper (George and Veeramani, 1994). The need of such modification was caused by the idea to apply "metric-type" function for the research of the structure of the set of infinite words as well as to study some other problems of words combinatorics. Since, in our opinion, fragmentary fuzzy pseudometrics are of their own interest, we first study basic properties fragmentary fuzzy pseudometrics as well as the (supra)topology they induce. In the last two sections we apply the above defined concepts and the obtained results to introduce a fragmentary fuzzy ultra pseudometric on the set of infinite words and to study the resulted structure.

The concept of a fragmentary fuzzy pseudometric, being a useful modification of the “ordinary" fuzzy pseudometric (George and Veeramani, 1994) is not its generalization since axiom (2FFPM) in the definition of a fragmentary fuzzy pseudometric contradicts axiom (2FPM) in the definition of a fuzzy pseudometric. As a possible direction for a further research we consider the introduction and the study of a "fuzzy metric-type" function that would contain both ordinary fuzzy pseudometrics and fragmentary fuzzy pseudometrics as special cases. The idea for the definition of such a function could be by replacing axiom (2FFPM) by the following more general axiom

$\left(2^{\varphi} \mathrm{FPM}\right) m(x, x, t)=\varphi(t) \geq m(x, y, t) \forall x, y \in X, \forall t \in \mathbb{R}^{+}$,

where $\varphi: \mathbb{R}^{+} \rightarrow[0,1]$ is a non-decreasing function such that $\lim _{t \rightarrow \infty} \varphi(t)=1$, and leaving the rest of the axioms (1FFPM), (3FFPM), (4FFPM), (5FFPM) unchanged. The function $m: X \times X \times \mathbb{R}^{+} \rightarrow(0,1]$ satisfying these conditions could be called a fuzzy $\varphi$ pseudometric and the corresponding pair $(X, m)$ a fuzzy $\varphi$-pseudometric space. Then one will gain "ordinary" fuzzy pseudometrics by taking $\varphi(t)=1$ for all $t \in \mathbb{R}^{+}$and fragmentary fuzzy metrics by taking $\varphi(t)=\frac{t}{1+t}$. Many other special examples could be obtained in such a way.

As a challenge for the future research we consider to undertake a deeper study of the properties of fuzzy $\varphi$-pseudometrics, in particular, of fragmentary fuzzy pseudometrics. As the first one of such properties to be studied we see completeness, complementations, the uniform structure, different types of continuous mappings of such spaces, in particular, contractions. It would be useful also to investigate the problem of the existence and the uniqueness of a fixed point for mappings of such spaces.

\section{References}

Allouche, J.-P., Shallit, J. (2003). Automatic sequences: Theory, applications, generalizations, Cambridge Univ. Press.

Afrouzi, G. A., Shakeri, S., Rasouli S. H., (2011). On the fuzzy metric spaces, The journal of Mathematics and Computer Science 2 (3), 475-482.

Calude, C. S., Jrgensen, H., Staiger, L. (2009). Topology on words.

Chang, C. L. (1968). Fuzzy topological spaces, J. Math. Anal. Appl., 24, 182-190.

Engelking, R. (1977). General Topology, Warszawa.

Frechet, M. (1906). Sur quelques points de calcul fonctionel, Rend Circ Mat. Palermo, 22, 1-74. 
George, A., Veeramani, P. (1994). On some results in fuzzy metric spaces, Fuzzy Sets Syst., 64, 395-399.

George, A., Veeramani, P. (1997). On some results of analysis for fuzzy metric spaces, Fuzzy Sets Syst., 90, 365-368.

Grecova, S., Šostak, A., Uljane. I. A construction of a fuzzy topology from a strong fuzzy metric A construction of a fuzzy topology from a strong fuzzy metric, , Applied General Topology, to appear.

Gregori, V., López-Crevillén, A., Morillas, S. (2009). On continuity and uniform continuity in fuzzy metric spaces, Proc. Work. App. Topology WiAT'09, 85-91.

Gregori, V. (2014). A survey of the theory of fuzzy metric spaces, Preprint.

Gregori, V., López-Crevillén, A., Morillas, S., Sapena, A. (2009). On convergence in fuzzy metric spaces, Topology and its Applications 156, 3002-3006.

Gregori, V., López-Crevillén, A., Morillas, S. (2009). On continuity and uniform continuity in fuzzy metric spaces, Proc. Work. App. Topology WiAT'09, 85-91.

Gregori, V., Miñana, J. J. (2013). Some concepts related to continuity in fuzzy metric spaces, Proceedings in the Conference in Applied Topology WiAT'13, 85-91.

Gregori, V., Morillas, S., Sapena, A. (2010). On a class of completable fuzzy metric spaces, Fuzzy Sets and Systems 161, 2193-2205.

Gregori, V., Romaguera, S. (2004). Characterizing completable fuzzy metric spaces, Fuzzy Sets and Systems 144, 411-420.

Höhle, U. (1980). Upper semicontinuous fuzzy sets and applications, J. Math. Anal. Appl., 78, 659-673.

Höhle, U. (1999). L-valued neighborhoods: Chapter 5 in Mathematics of Fuzzy Sets: Logic, Topology and Measure Theory, U. Höhle, S.E. Rodabaugh eds. - Handbook Series, vol.3, Kluwer Acad. Publ.

Kelly, J. L. (1955). General Topology, New York.

Klement, E. P., Mesiar, R., Pap, E. (2000). Triangular norms, Kluwer Acad. Publ.

Kramosil, I., Michalek, J. (1975). Fuzzy metrics and statistical metric spaces, Kybernetika 11, $336-344$.

Mardones-Perez, I., de Prada Vicente, M. A. (2015). Fuzzy pseudometric spaces vs fuzzifying structures, Fuzzy Sets and Syst., 267, 117-132.

Mardones-Perez, I., de Prada Vicente, M. A. (2012). A representation theorem for fuzzy pseudometrics, Fuzzy Sets and Syst., 195, 90-99.

Miñana, J. J., Šostak, A. Fuzzifying topology induced by a strong fuzzy metric, Fuzzy Sets and Systems, 6938 DOI information: 10.1016/j.fss.2015.11.005.

Menger, K. (1951). Probabilistic geometry, Proc. N.A.S. 27, 226-229.

Sapena, A. (2001). A contribution to the study of fuzzy metric spaces, Applied General Topology 2, 63-76.

Sapena, A., Morillas, S. (2009). On strong fuzzy metrics, Proc. Work. App. Topology WiAT’09, 135-141.

Schweizer, B., Sklar, A. (1960). Statisitcal metric spaces, Pacific J. Math. 10, 215-229.

Ying, M. S. (1991). A new approach to fuzzy topology, Part I, Fuzzy Sets and Syst., 39, 303-321.

Ying, M. S. (1992). A new approach to fuzzy topology, Part II, Fuzzy Sets and Syst., 47, 221-232.

Ying, M.S. (1993). A new approach to fuzzy topology, Part III, Fuzzy Sets and Syst., 55, 193-207.

Ying, M. S. (1993) Compactness in fuzzifying topology, Fuzzy Sets and Syst., 55, 79-92.

Yue, Y., Shi, F.-G. (2010). On fuzzy metric spaces, Fuzzy Sets and Syst., 161, 1105-1116.

Zhang, D., Xu, L. (1999). Categories isomorphic to FNS, Fuzzy Sets and Systems, 104, 373-380.

Zadeh, L. A. (1965). Fuzzy sets, Inform. and Control, 8, pp. 338-353.

Received August 31, 2016, accepted September 20, 2016 